\title{
ON HILBERT POLYNOMIAL OF CERTAIN DETERMINANTAL IDEALS
}

\author{
SHRINIVAS G. UDPIKAR \\ Department of Mathematics \\ S.P. Coll lege \\ Pune - 411030, India
}

(Received February 2, 1989 and in revised form July 10, 1989)

ABSTRACT. Let $x=\left(x_{i j}\right)$ be an $m(1)$ by $m(2)$ matrix whose entries $x_{i j}, 1<i<m(1)$, $1<j<m(2)$; are indeterminates over a field $k$. Let $K[X]$ be the polynomial ring in these $m(1) m(2)$ variables over $K$. A part of the second fundamental theorem of Invariant Theory says that the ideal $I[p+1]$ in $K[X]$, generated by $(p+1)$ by $(p+1)$ minors of $X$ is prime. More generally in [1], Abhyankar defines an ideal I[p,a] in $K[X]$, generated by different size minors of $X$ and not only proves its primeness but also calculates the Hilbert function as well as the Hilbert polynomial of this ideal. The said Hilbert polynomial is completely determined by certain integer valued functions $F_{D}(m, p, a)$. In this paper we prove some important properties of these integer valued functions.

KEY WORDS AND PHRASES. Standard bi-tableaux, monomials in minors, Hilbert polynomial, arithmetic genus, Irreducible varlety, recursive formula.

1980 AMS SUBJECT CLASSIFICATION CODE. 05A15, 13 B25.

\section{INTRODUCTION.}

We assume all notations and conventions of Abhyankar [1]. Let $\mathrm{Z}, \mathrm{N}, \mathrm{N}^{*}$ and $Q$ denote the set of all integers, nonnegative integers, positive integers and rational numbers respectively.

For any two integers $A$ and $B$, we let $[A, B]=\{D \in Z: A<D<B\}$.

For any $p \in N$, we denote by $Z(p), N(p)$, and $N^{\star}(p)$ the set of all maps from $[1, p]$ to $\mathrm{Z}, \mathrm{N}$ and $\mathrm{N*}$ respectively.

For any $p \in N^{*}$ and $D \in Z$, we let $Z(p, D)=\{d \varepsilon Z(p): d(1)+d(2)+\ldots+d(p)=D\}$ and $N(p, D)=\{d \varepsilon N(p): d(1)+d(2)+\ldots+d(p)=D\}$.

For any $p \in N$, we let $\operatorname{rec}(2, p)=\{(k, 1): k \varepsilon[1,2]$ and $1 \varepsilon[1, p]\}$.

For any $p \in N$, by a bi-vector of length $p$, we mean a map a: $\operatorname{rec}(2, p) \rightarrow N^{*}$, which for every $k \in[1,2]$ and $1 \in[1, p]$, associates $a(k, 1) \varepsilon N^{*}$ such that $a(k, 1)<a(k, 2)$ $<\ldots<a(k, p)$. In this case we put $\operatorname{len}(a)=p$.

By a bi-vector, we mean a bi-vector of length $p$ for some $p \in N$.

For any $p \in N, m \varepsilon N *(2)$ and a bi-vector a of length $p$, we say that a is bounded by $m$ and denote this by $a<m$, to mean that 


$$
a(k, i)<m(k) \text { for } k \in[1,2] \text { and } i \in[1, p] \text {. }
$$

Given any two bi-vectors $a$ and $a^{\prime}$ of lengths $p$ and $p^{\prime}$ respectively, we define $a<a^{\prime}$ to mean that $p>p^{\prime}$ and an $a(k, 1)<a^{\prime}(k, 1)$ for $k \varepsilon[1,2]$ and

$i \varepsilon\left[1, p^{\prime}\right]$. We note that this defines a partial order on the set vec(2) of all bivectors.

Given any $d \varepsilon N$, by a bi-tableau of depth $d$, we mean a mapping

$T:[1, d] \rightarrow \operatorname{vec}(2)$, which to every e $\varepsilon[1, d]$, associates $T[e] \varepsilon \operatorname{vec}(2)$.

A bi-tableau $T$ of depth $d$ is sald to be standard if len( $T[e])$ is positive for each $e \varepsilon[1, d]$ and

$$
\mathrm{T}[1]<\mathrm{T}[2]<\ldots<\mathrm{T}[\mathrm{d}] \text {. }
$$

Given any $m \varepsilon N^{*}(2), a \varepsilon \operatorname{vec}(2)$ and a bi-tableau $T$ of depth $d$, we say that

(i) $\mathrm{T}$ is bounded by $\mathrm{m}$ if $\mathrm{T}[\mathrm{e}]<\mathrm{m}$ for $\mathrm{e}=1,2, \ldots, \mathrm{d}$

(ii) $\mathrm{T}$ is predominated by $\mathrm{a}$ if $\mathrm{a}<\mathrm{T}[\mathrm{e}]$ for $\mathrm{e}=1,2, \ldots, \mathrm{d}$.

The area of a bi-tableau $T$ of depth $d$ is denoted by are( $T$ ) and is defined as

$$
\begin{array}{rlrl}
\operatorname{are}(T) & =\sum_{e=1}^{d} \operatorname{len}(T[e]) & \text { if } d \neq 0 \text { and } \\
& =\quad 0 \quad \text { if } d=0 .
\end{array}
$$

Finally for any element $V$ in an overring of $Q$ and any integer $r$, we set

$$
\left(\begin{array}{l}
v \\
r
\end{array}\right)=\left\{\begin{array}{cl}
\frac{v(v-1) \ldots(v-r+1)}{r !} & \text { if } r>0 \\
0 & \text { if } r<0
\end{array}\right.
$$

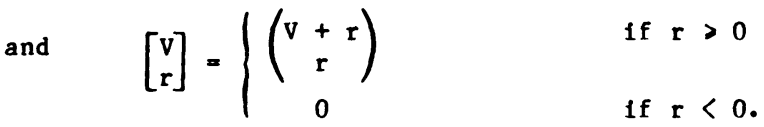

Let $x=\left(x_{1 j}\right)$ be any $m(1)$ by $m(2)$ matrix whose entries $x_{1 j}, 1 \leqslant 1 \leqslant m(1)$, $1<j<m(2)$, are indeterminates over a field $K$. Let $K[X]$ be the polynomial ring in these $\mathrm{m}(1) \mathrm{m}(2)$ variables. Clearly to every bi-vector a of length $\mathrm{p}$ and bounded by $\mathrm{m}$, there corresponds a uniquely determined $p \times p$ minor of $x$, say mor $(x, a)$, formed by the rows with row numbers $a(1,1), a(1,2), \ldots, a(1, p)$ and by the columns with column numbers $a(2,1), a(2,2), \ldots, a(2, p)$. By convention we define $\operatorname{mor}(X, a)=1$ if $\operatorname{len}(a)=0$.

Further, to a bi-tableau $T$ of depth $d$, there corresponds a product of minors of $X$, which we call a monomial in minors of $X$. A monomial in minors of $X$ is said to be standard if the corresponding bi-tableau is standard.

Using straightening formula, DeConcin1, Eisenbud, and Procesi [2], proved that the standard monomials in minors of $X$ form a base for the vector space $K[x]$ over $K$. Abhyankar proves this by enumerating the number of standard bi-tableaux with certain conditions. In fact, Abhyankar [3] proves that the number of standard bi-tableaux of area $v$, bounded by $m$ and predominated by a given fixed bi-vector a of length $p$ is equal to

$$
H(V)={ }_{D \varepsilon Z}(-1) D_{F_{D}}(m, p, a) \quad\left[\begin{array}{c}
V \\
C-D
\end{array}\right]
$$


where $C=C[m, p, a]=\sum_{i=1}^{p}(m(1)-a(1, i))+\sum_{i=1}^{p}(m(2)-a(2, i))+p-1$

and $F_{D}(m, p, a), D \in Z$, are integer valued functins defined in section 2 .

He further proves that if a is a given bi-vector of length $p$, bounded by $m$ and if $I[p, a]$ is the ideal in $K[X]$ generated by all minors of $X$ corresponding $t$ : the bivectors $b$ not predominated by $a$, then $I[p, a]$ is a homogeneous prime ideal in $K[X]$ and $H(V)$ is the Hilbert function as well as the Hilbert polynomial of $I[p, a]$ in $K[X]$. In particular, if $a(k, i)=i$ for $k \varepsilon[1,2]$ and $i \varepsilon[1, p]$, then $I[p, a]$ is the ideal in $K[X]$ generated by $(p+1)$ by $(p+1)$ minors of $X$ and hence it follows by the above theorem that $i t$ is a prime ideal in $K[X]$. This forms a part of the second fundamental theorem of invariant theory and was originally proved by Pascal in 1888 and then reproved by Mount [4], Eagon and Hochster [5] and others.

Now $H(V)$ is a polynomial in $V$ with rational coefficients and of degree $C$. Further $C$ ! times the coefficient of $V^{C}$ in $H(V)$ is $F_{0}(m, p, a)$, which equals the order of the irreducible variety defined by $I[p, a]$ in the $(m(1) m(2)-1)$ dimensional projective space over $K$ (Refer to remark (20.18) of [1]). From this it follows that $F_{0}(m, p, a)$ is a positive integer (see theorem (2.1) of section 2). Further arithmetic genus of $I[p, a]$ is $(-1)^{C}\left[\left(F_{0}-F_{1}+F_{2}-\ldots+(-1)^{C} F_{C}\right)-1\right]=0$. Thus $F_{D}(m, p, a), D \varepsilon z$ determine important geometric characters of the varlety defined by $I[p, a]$ from Zariski and Samuel [6]. Hence $1 t$ is interesting to study the properties of $F_{D}(m, p, a), D \varepsilon z$.

2. INTEgER VALUED FUNCTIONS $F_{D}(m, p, a)$.

For any $m \varepsilon N^{*}(2), p \varepsilon N^{*}$, we put $\operatorname{vec}(2)=$ the set of all bi-vectors, vec: $[2, p]=$ the set of all bi-vectors of length $p$ and $\operatorname{vec}(2, m, p)=\{a \varepsilon \operatorname{vec}[2, p]: a<m\}$.

Let $\mathrm{m} \varepsilon \mathrm{N}^{*}(2), \mathrm{p} \varepsilon \mathrm{N}^{*}, a \varepsilon \operatorname{vec}(2, \mathrm{~m}, \mathrm{p})$ and $\mathrm{k} \varepsilon[1,2]$. Let $\mathrm{k}^{\prime}=2$ if $k=1$ and $k^{\prime}=1$ if $k=2$.

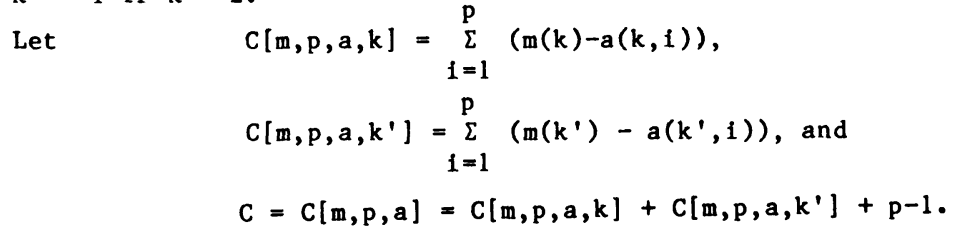

We now define integer valued functions $F_{D}(m, p, a), D \in Z$.

For every $1, j \in[1, p]$, e $\varepsilon Z(p)$ we denote by $G(m, p, a, e)$, the $p \times p$ matrix whose $(i, j)^{\text {th }}$ entry is $G_{i j}(m, p, a, e)=\left(\begin{array}{c}m(k)-a(k, 1)+i-j \\ m(k)-a(k, 1)-e_{1}\end{array}\right)\left(\begin{array}{c}m\left(k^{\prime}\right)-a\left(k^{\prime}, j\right)+j-i \\ e_{1}\end{array}\right)$ where $e_{i}=e(1)$ for $1=1,2, \ldots, p$. Further let for every $E \in Z$, $H_{E}(m, p, a)=\sum_{e \varepsilon Z(p, E)} \operatorname{det} G(m, p, a, e)$. Then for every $D \varepsilon Z$, we define $F_{D}(m, p, a)=\sum_{E \varepsilon Z}\left(\begin{array}{l}E \\ D\end{array}\right) \quad H_{E}(m, p, a)$

In view of the usual properties of binomial coefficients and determinants, we may assume that $0<e_{i}<(m(k)-a(k, 1))$ for $1=1,2, \ldots, p$. Hence the above sum is 
essentially finite.

In [1], Ahymankir proves the following:

THEOREM 2.1. Let there be given any $m \in N^{*}(2), p \leqslant N^{*}$ and a $\varepsilon$ vec $(2, m, p)$. Then we have

(i) $\quad F_{i}(m, p, a)$ is a positive integer,

(ii) for all $\mathrm{D} \in \mathrm{Z}, \mathrm{F}_{\mathrm{D}}(\mathrm{m}, \mathrm{p}, \mathrm{a})$ is a nomnegat ive integer and

(iii) $\quad\left\{D=Z: F_{D}(m, p, a) \neq 0\right\} \subseteq[0, C(m, p, a)]$ where

$C(m, p, a)=\min \left\{C[m, p, a, k], C\left[m, p, a, k^{\prime}\right]\right\}$.

PROOF. Refer to Leinma 9.15 and Theoreins $9.13,9.17$ of [1].

He further proposes the following problems. Given any $m \varepsilon N^{*}(2), p \varepsilon N^{*}$ and a $\varepsilon \operatorname{vec}(2, m, p)$, does there exist a nonnegative integer $c^{\star}(m, p, a)$ such that

Problem (I) $\left\{D \in Z: F_{D}(m, p, a) \neq 0\right\}=\left[0, C^{*}(m, p, a)\right]$ ?

Problem (II) If so, then is it possible that

$$
C^{\star}(m, p, a)=C(m, p, a) ?
$$

(Refer to remark 9.18 of [1]).

This paper settles both these problems in an affirmative manner.

3. THF: MAIN RESULT.

We follow the usual convention that the sum over an empty family is zero. For any $p \in N$ and $A \in Z$, we let $J(p)=$ all subsets of $[1, p]$ and $J(p, A)=$ all subsets of $[1, \mathrm{p}]$ with cardinality $A$.

Let there be given any $m \varepsilon N^{*}(2), p \varepsilon N^{*}, b \varepsilon \operatorname{vec}[2, p+1], u \in J(P), U \varepsilon[0, p]$ and $k \varepsilon[1,2]$. Further let

$$
k^{\prime}= \begin{cases}2 & \text { if } k=1 \\ 1 & \text { if } k=2\end{cases}
$$

We define the sets $M[p, b, k, u]$ and $M(p, b, k, U)$ as follows:

$$
M[p, b, k, u]=\left\{\begin{array}{c}
a \varepsilon \operatorname{vec}[2, p]: a\left(k, 1^{*}\right)=b\left(k, i^{*}\right) \text { for all } i^{*} \varepsilon[1, p], \\
a\left(k^{\prime}, 1\right) \varepsilon\left[b\left(k^{\prime}, 1\right)+1, b\left(k^{\prime}, 1+1\right)-1\right], \text { for all } 1 \varepsilon u \\
\text { and } a\left(k^{\prime}, j\right)=b\left(k^{\prime}, j\right), \text { for all } j \varepsilon[1, p] \backslash u
\end{array}\right\}
$$

and $M(p, b, k, U)=U M[p, b, k, u], u \in J(p, U)$

Given any $m \varepsilon N^{*}(2), p \varepsilon N^{*}, b \varepsilon \operatorname{vec}[2, p+1]$ and $k \varepsilon[1,2]$, let $m^{\star} \varepsilon N^{*}(2)$ be such that

(1) $m^{*}\left(k^{\prime}\right)=m\left(k^{\prime}\right)$ and $m^{*}(k)=m(k)+1$ holds. Further let $p^{*} \varepsilon N^{*}$ and

$$
a^{*} \varepsilon \operatorname{vec}\left(2, m^{*}, p^{*}\right) \text { be such that either }
$$

(2a) $p^{*}=p$ and $m^{*}(k)-a^{*}\left(k, p^{*}\right) \neq 0$ and $a^{*}(\hat{k}, \hat{1})=b(\hat{k}, \hat{1})$ for all $\hat{k} \varepsilon[1,2]$ and

$1 \varepsilon[1, p]$ and $b(\hat{k}, p+1)=m(\hat{k})+1$ for all $\hat{k} \varepsilon[1,2]$ holds or

(2b) $p^{*}=p+1, m^{*}(k)-a^{*}\left(k, p^{*}\right)=0$ and $a^{*}=b$ holds.

Finally for every $m \varepsilon N^{*}(2), p \varepsilon N^{*}, a \varepsilon \operatorname{vec}[2, p], U \varepsilon[0, p]$ and $D \varepsilon Z$, we 
set

$$
S_{U}(m, p, a, D)=\sum_{I} \sum_{[0, U]}^{U}\left(F_{D-I}(m, p, a)\right.
$$

We now state a useful recursive formula in the following.

THEOREM 3.1. Let there be given any $\mathrm{m} \varepsilon \mathrm{N}^{*}(2), \underset{*}{\mathrm{p}} \varepsilon \mathrm{N}^{*}, \mathrm{k} \varepsilon[1,2]$, $\mathrm{b} \varepsilon \operatorname{vec}[2, \mathrm{p}+1]$ and $\mathrm{m}^{*} \varepsilon \mathrm{N}^{*}(2), \mathrm{p}^{*} \varepsilon \mathrm{N}^{*}, \mathrm{a}^{*} \varepsilon \operatorname{vec}\left(2, \mathrm{~m}^{*}, \mathrm{p}^{*}\right)$ such that (1) holds and either (2a) or (2b) holds. Then for every $D \varepsilon Z$, we have

$F_{D}\left(m^{*}, p^{*}, a^{*}\right)=\sum_{\varepsilon}[0, p] \cup \sum_{\varepsilon}[0, p] a \sum_{M(p, b, k, U)}\left(\sum_{I}^{U}\right) F_{D-I}(m, p, a)$.

PROOF. Refer to Theorem (9.8*), section 9 of [1].

In the above recursive formula, by interchanging the summations over $I$ and $U$ and by noting the fact that $\left(\begin{array}{l}U \\ I\end{array}\right)=0$ if $I>U>0$, we see that

$$
F_{D}\left(m^{*}, p^{*}, a^{*}\right)=\sum_{U \in[0, p]} \sum_{a \in M(p, b, k, U)} S_{U}(m, p, a ; D)
$$

We shall use this form of the recursive formula later in this section.

In what follows, we shall prove some results which enable one to answer the first problem posed by Abhyankar, in an affirmative manner [Refer to problem (I) at the end of section 2 ].

THEOREM 3.2. Let $k \in[1,2], p \in N^{*}$ and $b \in \operatorname{vec}[2, p+1]$ be given. Then we have the following.

(I) $M(p, b, k, 0)=M[p, b, k, \phi]$ is a nonempty set consisting of the unique element $\tilde{b} \varepsilon \operatorname{vec}[2, p]$ obtained from b by putting

$$
\tilde{b}(\hat{k}, i)=b(\hat{k}, i) \text { for } a 11 \hat{k} \varepsilon[1,2] \text { and } i \varepsilon[1, p] \text {. }
$$

(II) If $M(p, b, k, 1)=\phi$ then $\left\{b\left(k^{\prime}, j\right)\right\}, j=1,2, \ldots p$, is a sequence of consecutive integers i.e. $b\left(k^{\prime}, j+1\right)=b\left(k^{\prime}, j\right)+1$, for all $j \in[1, p-1]$ and consequent $1 y$

$M(p, b, k, U)=\phi$ for every $U>1$.

PROOF. (I) Since $\phi$ is the on $1 y$ subset of $[1, p]$ of cardinality zero, we have $M(p, b, k, 0)=M[p, b, k, \phi]$. The rest follows from the definition of $M[p, b, k, \phi]$. Let $u_{i}=\{1\}$ where 1 is an arbitrarily chosen element of $[1, p-1]$. Then by definition of $\mu\left[p, b, k_{1}, u_{1}\right]$ we have,

$$
\begin{aligned}
& M\left[p, b, k, u_{1}\right]=\phi \Leftrightarrow\left\{b\left(k^{\prime}, 1\right)+1, b\left(k^{\prime}, 1+1\right)-1\right]=\phi \\
& \Leftrightarrow b\left(k^{\prime}, i+1\right)=b\left(k^{\prime}, 1\right)+1, \text { so that }\left\{b\left(k^{\prime}, j\right)\right\},
\end{aligned}
$$

$j=1,2, \ldots p$, is an increasing sequence of consecutive integers.

Finally, if $U>1$ and $u \varepsilon J(p)$ is any set of cardinaliy $U$, then there is an $i \varepsilon u$ such that $i \varepsilon[1, p-1]$. Now if a $\varepsilon M(p, b, k, U)$, then we must have $a\left(k^{\prime}, i\right) \varepsilon\left[b\left(k^{\prime}, i\right)+1, b\left(k^{\prime}, i+1\right)-1\right]$, which is a contradiction since $\left\{b\left(k^{\prime}, j\right)\right\}$, $j=1,2, \ldots . p$, is a sequence of consecutive integers. Hence $M(p, b, k, U)=\phi$ for every $U>1$ if $M(p, b, k, 1)=\phi$. Hence the theorem.

LEMMA 3.1. Let $m \in N^{*}(2), p \in N^{*}, b \varepsilon \operatorname{vec}[2, p+1]$ and $k \varepsilon[1,2]$ be given. Let $\mathrm{m}^{*} \varepsilon \mathrm{N}^{*}(2), \mathrm{p}^{*} \varepsilon \mathrm{N}^{*}$ and $\mathrm{a}^{*} \varepsilon \operatorname{vec}\left(2, \mathrm{~m}^{*}, \mathrm{p}^{*}\right)$ be such that (1) is satisfied and either $(2 a)$ or $(2 b)$ is satisfied. Let $\tilde{b}$ be the unique element of vec $[2, p]$ obtained $f$ rom $b$ by putting $\tilde{b}(\hat{k}, \hat{i})=b(\hat{k}, \hat{i})$ for $a 11 \hat{k} \in[1,2]$ and $i \varepsilon[1, p]$. 


$$
\begin{aligned}
& F_{1}\left(. *^{*}, p^{*}, a^{*}\right)=0 \Rightarrow F_{1}(m, p, \tilde{b})=0 \text { and } \\
& \qquad F_{1+j}\left(m^{*}, p^{*}, a^{*}\right)=F_{1+j}(m, p, \tilde{b}) \text { for every } j \varepsilon N .
\end{aligned}
$$

PROOF. In view of theorem (3.1) and (I) of theurem (3.2), we have

$$
\begin{aligned}
F_{1}\left(m^{*}, p^{*}, a^{*}\right)=F_{1}(m, p, \tilde{b}) & +\sum_{a \varepsilon M(p, b, k, l)}\left[F_{1}(m, p, a)+F_{0}(m, p, a)\right] \\
& +\sum_{U \varepsilon[2, p]} \quad \sum_{a \in M(p, b, k, U)} S_{U}(m, p, a ; 1) .
\end{aligned}
$$

But by theorem (2.1) of section 2 , we have $F_{0}(m, p, a)>0$ and $F_{D}(m, p, a)>0$ for every $D \in Z$. Hence $F_{1}\left(m^{*}, p^{*}, a^{*}\right)=0 \Rightarrow F_{1}(m, p, \tilde{b})=0$ and $M(p, b, k, 1)=\phi$. But then by (II) of theorem (3.2), we have $M(p, b, k, U)=\phi$ for every $U>2$. Hence given any

$j \varepsilon N$, replacing $l$ by $1+j$ in the above formula we get

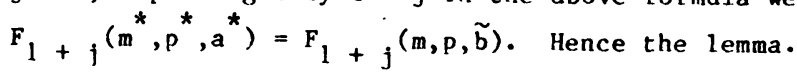

LEMMA 3.2. Let $D \in \mathrm{N}^{*}$. Assume that for all $\mathrm{m} \varepsilon \mathrm{N}^{*}(2), p \varepsilon \mathrm{N}^{*}$, $b \in \operatorname{vec}[2, p+1], k \in[1,2]$ and for all $\mathrm{m}^{*} \varepsilon \mathrm{N}^{*}(2), \mathrm{p}^{*} \varepsilon \mathrm{N}^{*}$ and $\mathrm{a}^{*} \varepsilon \operatorname{vec}\left(2, \mathrm{~m}^{*}, \mathrm{p}^{*}\right)$ satisfying (1) and satisfying (2a) or (2b), we have

$$
\begin{aligned}
F_{D}\left(m^{*}, p^{*}, a^{*}\right)=0= & (i) F_{D}(m, p, \tilde{b})=0 \text { and } \\
& \text { (ii) } F_{D+j}\left(m^{*}, p^{\star}, a^{*}\right)=F_{D+j}(m, p, \tilde{b}) \text { for all } j \varepsilon N \text {. }
\end{aligned}
$$

Then for every a $\varepsilon \operatorname{vec}(2, m, p)$, we have

$$
F_{D}(m, p, a)=0 \Rightarrow F_{D+j}(m, p, a)=0 \text { for every } j \in N \text {. }
$$

PROOF. We prove this by double induction on $p$ and $r(p)=m(k)-a(k, p)$. Suppose that for $a^{*} \varepsilon \operatorname{vec}\left(2, m^{*}, p^{*}\right), F_{D}\left(m^{*}, p^{*}, a^{*}\right)=0$. Hence by assumption, (i) and (ii) hold. If $r^{*}\left(p^{*}\right)=m^{*}(k)-a^{*}\left(k, p^{*}\right) \neq 0$, then $p=p^{*}$ so that $(2 a)$ holds and $r(p)<r^{*}\left(p^{*}\right)$. Hence by $(i)$ and inductive hypothesis, we have $F_{D+j}(m, p, \tilde{b})=0$, for all $j \in N$.

Hence by (ii) $F_{D+j}\left(m^{*}, p^{*}, a^{*}\right)=0$ for all $j \varepsilon N$. On the other hand if $r^{*}\left(p^{*}\right)=0$ and $p^{*}$ $=p+1$, then (2b) holds and as above, we get

$F_{D+j}\left(m^{*}, p^{*}, a^{\star}\right)=0$, for all $j \varepsilon N$.

Thus to complete the proof, we only need to prove the result when $p=1$ and $r(p)$ $=m(k)-a(k, p)=0$. But in this case $c(m, p, a)=0$ and hence by theorem $(2.1)$, we see that $F_{D+j}(m, p, a)=0$, for all $D \in N^{*}$ and $j \in N$. Hence the lemma.

Now we are in a position to prove the main result of this section.

THEOREM 3.3. For every $D, j \in N^{*}$, we have the following:

$F_{D}(m, p, a)=0 \Rightarrow F_{D+j}(m, p, a)=0$, for every triplet $(m, p, a)$ such that $m \varepsilon N^{*}(2)$, $p \varepsilon N^{*}$ and $a \varepsilon \operatorname{vec}(2, m, p)$.

PROOF. By induction on D. In view of lemma 3.1 and lemma 3.2 , the theorem is clearly true if $D=1$. Now assume that $D$ is any integer greater than 1 and the theorem holds for every $D^{\prime} \varepsilon N^{*}$ such that $D^{\prime}<D$. To prove the theorem for this fixed $D$, we shall use lemma 3.2 .

So let there be given $m \in N^{*}(2), p \in N^{*}, b \varepsilon \operatorname{vec}[2, p+1], k \varepsilon[1,2]$ and $\mathrm{m}^{\star} \varepsilon \mathrm{N}^{\star}(2), \mathrm{p}^{*} \varepsilon \mathrm{N}^{*}, \mathrm{a}^{*} \varepsilon \operatorname{vec}\left(2, \mathrm{~m}^{*}, \mathrm{p}^{*}\right)$ such that (1) is satisfied and (2a) or (2b) is 
satisfied.

$$
\begin{aligned}
& \text { Assume that } F_{D}\left(m^{*}, p^{*}, a^{*}\right)=0 \text {. By theorem 3.1, we have } \\
& F_{D}\left(m^{*}, p^{*}, a^{*}\right)=F_{D}(m, p, \tilde{b})+\underset{U E[1, p]}{i} \underset{a \in M(p, b, k, U)}{\sum} S_{U}(m, p, a ; D)=0
\end{aligned}
$$

Note that $F_{D}(m, p, a) \geqslant 0$ for every $D \in Z$. Hence, if for some $U \in[1, p]$, $M(p, b, k, U)$ is nonempty, then by $(3.1)$, we must necessarlly have $S_{U}(m, p, a ; D)=0$. Hence by definition of $\mathrm{S}_{U}(\mathrm{~m}, \mathrm{p}, \mathrm{a} ; \mathrm{D})$ and the fact that $\left(\begin{array}{l}U \\ l\end{array}\right)>0$ if $\mathrm{I} \varepsilon[0, U]$, we have $F_{D-I}(m, p, a)=0$ for every $I \varepsilon[0, U]$. In particular, $F_{D-U}(m, p, a)=0$. But $U>1$. Hence by inductive hypothesis, we get $F_{D+j-U}(m, p, a)=0$ for every $j \varepsilon N$. Hence, given any $j \varepsilon N$, by using the above identity as $j$ is successively replaced by $j+U-I \varepsilon N$, as $I$ varies from 0 to $U$, we get $S_{U}(m, p, a ; D+j)=0$. Thus for $U>1$, in view of $(3.1)$, we have either $M(p, b, k, U)=\phi$ or $S_{U}(m, p, a ; D+j)=0$, for all j $\varepsilon$.

Hence by recursive formula, we get $F_{D+j}\left(m^{*}, p^{*}, a^{*}\right)=F_{D+j}(m, p, \tilde{b})$ for all $j \varepsilon N$. But by $(3.1)$, we certainly have $F_{D}(m, p, \tilde{b})=0$.

Hence by Lemma 3.2 , we get the required result.

From this theorem, it is clear that, given any $m \varepsilon N^{*}(2), p \in N^{*}$ and a $\varepsilon \operatorname{vec}(2, m, p)$, the set $\left\{D \in Z: F_{D}(m, p, a) \neq 0\right\}$ is an interval of the form $\left[0, c^{\star}(m, p, a)\right]$ for some nonegative integer $C^{\star}(m, p, a)$.

Thus, Abhyankar's conjecture is true.

4. SOLUTION TO SECOND PROBLEM.

From section 2, we know that for every $D \varepsilon Z$.

$$
F_{D}(m, p, a)=\sum_{E \in Z}\left(\begin{array}{l}
E \\
D
\end{array}\right) \sum_{\varepsilon Z(p, E)} \quad \operatorname{det}\left\{\left(\begin{array}{c}
m(k)-a(k, i)+1-j \\
m(k)-a(k, i)-e_{i}
\end{array}\right)\left(\begin{array}{c}
m\left(k^{\prime}\right)-a\left(k^{\prime}, j\right)+j-i \\
e_{i}
\end{array}\right)\right\}
$$

where $m \varepsilon N^{*}(2), p \varepsilon N^{*}, a \varepsilon \operatorname{vec}(2, m, p), k \varepsilon[1,2]$ and

$$
k^{\prime}= \begin{cases}2 & \text { if } k=1 \\ 1 & \text { if } k=2\end{cases}
$$

Noting that $0<e_{1}<(m(k)-a(k, i))$ for $1=1,2, \ldots, p$; we see that $F_{D}(m, p, a)=\operatorname{det}\left\{\left(\begin{array}{l}m\left(k^{\prime}\right)-a\left(k^{\prime}, j\right)+j-1 \\ m(k)-a(k, 1)\end{array}\right)\right\}$

if $D=C[m, p, a, k]=\sum_{i=1}^{p}(m(k)-a(k, i))$.

Hence in particular, $F_{D}(m, p, a)=1$, if no two of $\left(m\left(k^{\prime}\right)-a\left(k^{\prime}, j\right)\right), j=1,2, \ldots, p$ are consecutive and $\left(m\left(k^{\prime}\right)-a\left(k^{\prime}, j\right)\right)=(m(k)-a(k, j))$ for $j=1,2, \ldots, p$; where $D=C[m, p, a, k]$.

Hence by the main theorem of section 3 , we see that $F_{D}(m, p, a)>0$ for every $D \in[0, C(m, p, a)]$ where $C(m, p, a)=\min \left\{c[m, p, a, k], C\left[m, p, a, k^{\prime}\right]\right\}$. 
Hence there exist $m \varepsilon N^{\star}(2), p \in N^{\star}$ and a $\varepsilon \operatorname{vec}(2, m, p)$ such that $F_{D}(m, p, a)>0$ for every $D \in[0, C(m, p, a)]$.

Affirmative answers to problems (I) and (II) enable us to write the Hilbert Polynomial of $I[P, a]$ in $K[X]$ in much tidier form as follows:

$$
H(V)=\sum_{D=0}^{C^{*}(m, p, a)}(-1)^{D} F_{D}(m, p, a)\left[\begin{array}{c}
V \\
C-D
\end{array}\right]
$$

where $C^{\star}(m, p, a)$ is some integer such that $0<C^{\star}(m, p, a)<C(m, p, a)$ and $F_{D}(m, p, a)>0$ for every $D \in\left[0, c^{\star}(m, p, a)\right]$.

ACKNOWLEDGEMENTS. I wish to thank Professor Shreeram S. Abhyankar for his valuable guidance while working with him for my Ph.D Degree. I wish also to thank Panjab University, Chandigarh for the award of the U.G.C. fellowship and Bhaskaracharya Pratishthana, Pune, for providing necessary facilities.

\section{REFERENCES}

1. ABHYANKAR, S., Enumerative Combinatorics of Young Tableaux, Marcel Dekker, Inc. 1988.

2. DECONCINI, EISENBUD, PROCESI, Yong Diagrams and Determinantal Varietles; Inventiones Mathematicae 56, (1980), 129-165

3. ABHYANKAR, S., Combinatorie des tableaux de Young, varietes determinantielles et calcul de fonctions de Hilbert; Nice lecture notes by A. Galligo; Universita E. Polytecnico di Torino: Rendiconti del Seminario Matematico 1985.

4. MOUNT, K.R., A Remark on Determinantal Loc1; Journal London Math. Soc. 42, (1967), 595-598.

5. EAGON, J. and HOCHSTER, M., Cohen Macaulay Rings, Invariant Theory and the Generic Perfection of Determinantal Loc1; American J. Math. 93, (1971), 10201058 .

6. ZARISKI, 0 and SAMUEL, P., Commutative Algebra, Volume II, Van Nostrand Princeton 1969. 


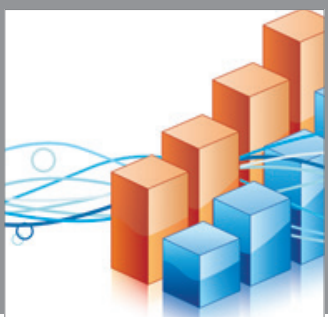

Advances in

Operations Research

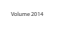

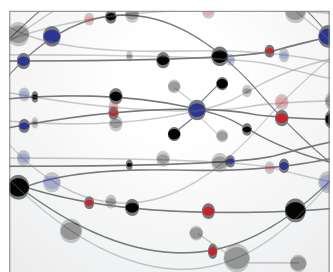

\section{The Scientific} World Journal
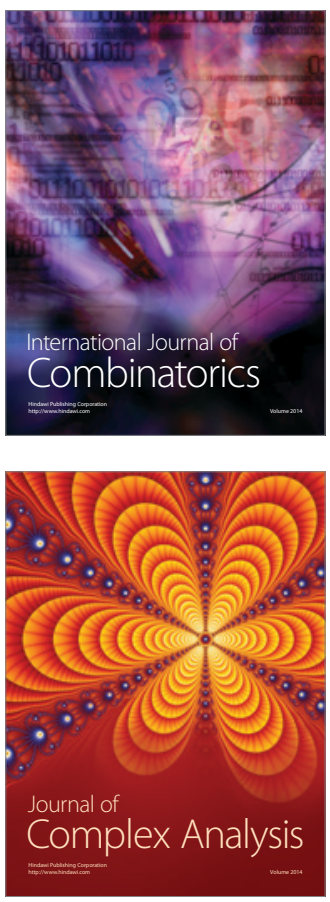

International Journal of

Mathematics and

Mathematical

Sciences
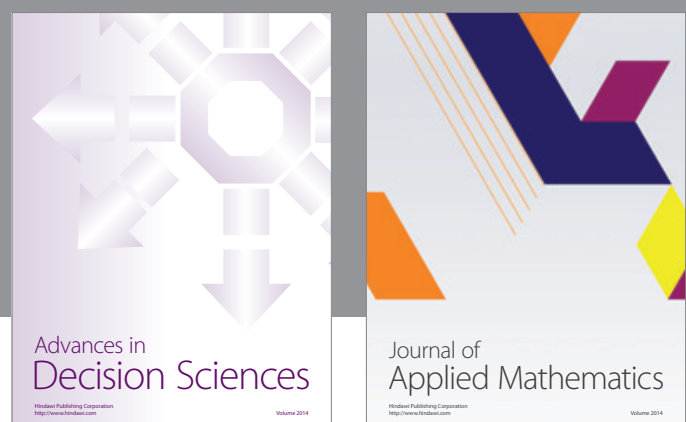

Journal of

Applied Mathematics
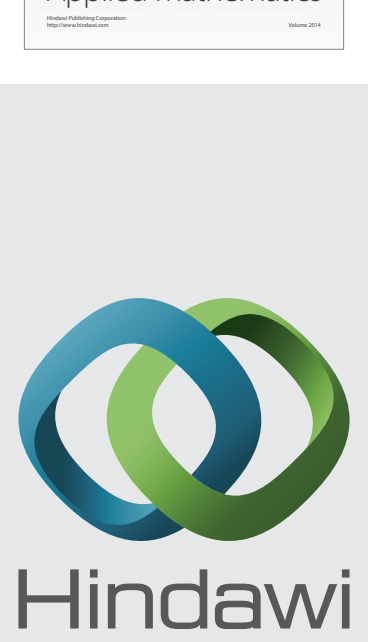

Submit your manuscripts at http://www.hindawi.com
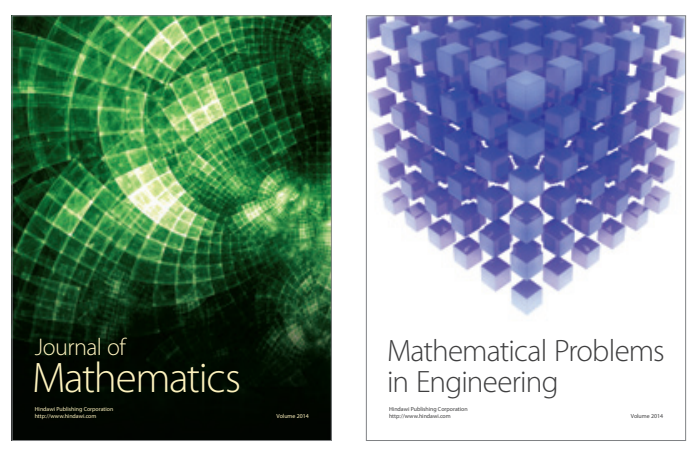

Mathematical Problems in Engineering
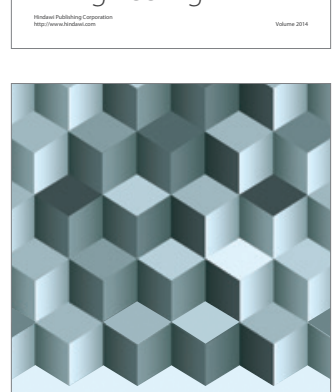

Journal of

Function Spaces
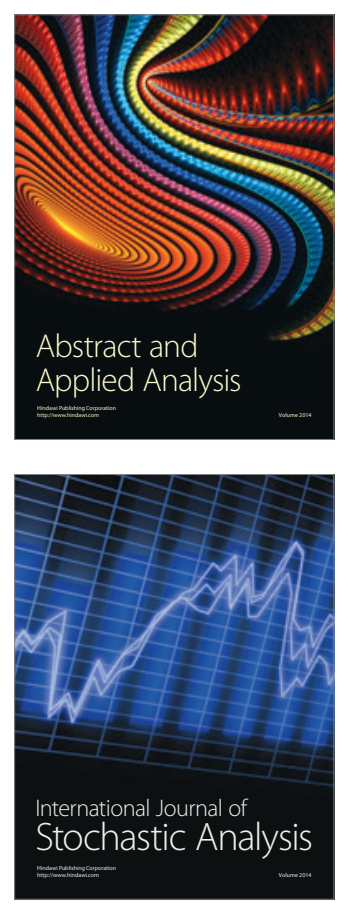

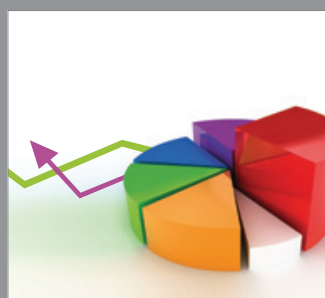

ournal of

Probability and Statistics

Promensencen
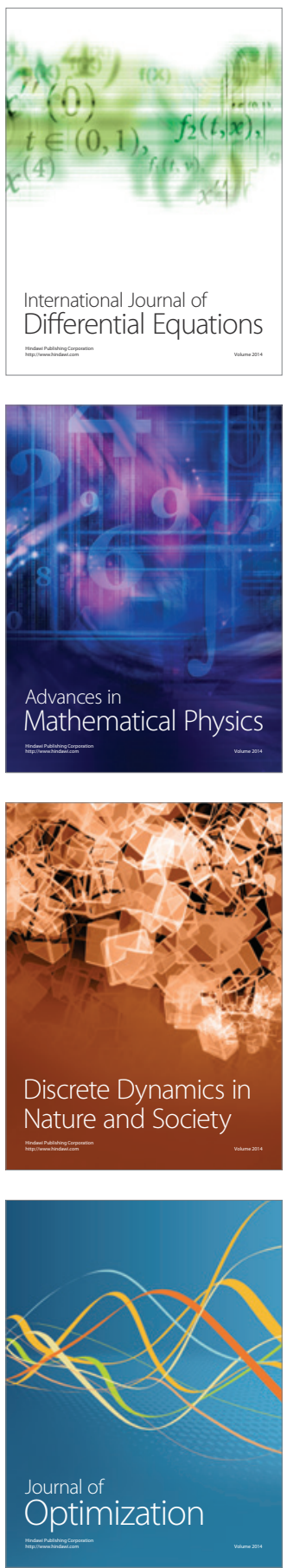